\title{
Magnetized Dusty Black Holes and Wormholes
}

\author{
Kirill A. Bronnikov $1,2,3, *$ (i) Pavel E. Kashargin ${ }^{4}$ and Sergey V. Sushkov ${ }^{4, *(\mathbb{C})}$ \\ 1 Center of Gravitation and Fundamental Metrology, VNIIMS, Ozyornaya Ul. 46, 119361 Moscow, Russia \\ 2 Institute of Gravitation and Cosmology, Peoples' Friendship University of Russia (RUDN University), \\ Miklukho-Maklaya 6, 117198 Moscow, Russia \\ 3 Elementary Particle Physics Department, National Research Nuclear University "MEPhI", \\ Kashirskoe Shosse 31, 115409 Moscow, Russia \\ 4 Institute of Physics, Kazan Federal University, Kremliovskaya St. 16a, 420008 Kazan, Russia; \\ pkashargin@mail.ru \\ * Correspondence: kb20@yandex.ru (K.A.B.); sergey_sushkov@mail.ru (S.V.S.)
}

check for updates

Citation: Bronnikov, K.A.; Kashargin, P.E.; Sushkov, S.V. Magnetized Dusty Black Holes and Wormholes. Universe 2021, 7, 419. https://doi.org/ $10.3390 /$ universe7110419

Academic Editor: Gonzalo J. Olmo

Received: 26 September 2021

Accepted: 29 October 2021

Published: 3 November 2021

Publisher's Note: MDPI stays neutral with regard to jurisdictional claims in published maps and institutional affiliations.

\section{Copyright: (c) 2021 by the authors.} Licensee MDPI, Basel, Switzerland. This article is an open access article distributed under the terms and conditions of the Creative Commons Attribution (CC BY) license (https:/ / creativecommons.org/licenses/by/ $4.0 /)$.

\begin{abstract}
We consider the generalized Tolman solution of general relativity, describing the evolution of a spherical dust cloud in the presence of an external electric or magnetic field. The solution contains three arbitrary functions $f(R), F(R)$ and $\tau_{0}(R)$, where $R$ is a radial coordinate in the comoving reference frame. The solution splits into three branches corresponding to hyperbolic $(f>0)$, parabolic $(f=0)$ and elliptic $(f<0)$ types of motion. In such models, we study the possible existence of wormhole throats defined as spheres of minimum radius at a fixed time instant, and prove the existence of throats in the elliptic branch under certain conditions imposed on the arbitrary functions. It is further shown that the normal to a throat is a timelike vector (except for the instant of maximum expansion, when this vector is null), hence a throat is in general located in a T-region of space-time. Thus, if such a dust cloud is placed between two empty (Reissner-Nordström or Schwarzschild) space-time regions, the whole configuration is a black hole rather than a wormhole. However, dust clouds with throats can be inscribed into closed isotropic cosmological models filled with dust to form wormholes which exist for a finite period of time and experience expansion and contraction together with the corresponding cosmology. Explicit examples and numerical estimates are presented. The possible traversability of wormhole-like evolving dust layers is established by a numerical study of radial null geodesics.
\end{abstract}

Keywords: wormholes; black holes; dustlike matter; collapse; Tolman's solution; general relativity

\section{Introduction}

A wormhole is a kind of spatial geometry resembling a tunnel that connects two different regions of the same space-time, or two different space-times. Such geometries as solutions to the gravitational field equations were first mentioned in [1-4], but they were not traversable in the sense that a subluminal particle could not travel from one "end of the tunnel" to the other and return back. Probably the first exact traversable wormhole solutions were discussed in [5,6] (1973) in the Einstein-scalar theory in which the scalar is of phantom nature, i.e., has a wrong sign of the kinetic term in the Lagrangian. Later, an evolving version of this scalar-vacuum solution was found [7]. One can also mention G. Clement's papers [8,9] describing static higher-dimensional wormholes. However, the greatest interest in these objects arose after the work of M. Morris and K. Thorne [10] (1988) who showed that, in the framework of Einstein gravity, maintaining a static wormhole throat needs "exotic" matter that violates the Null Energy Condition (NEC). Overviews of wormhole research can be found, for example, in [11,12].

Wormholes have been considered in various theories of gravity and diverse models of matter as a source of the geometry. In particular, K. Bronnikov [5] presented static, spherically symmetric wormhole solutions with or without an electric charge both in general relativity (GR) and a class of scalar-tensor theories. Refs. [13-16] describe wormholes 
with electric or magnetic charges in the Einstein-Maxwell-dilaton theory. A family of dynamic solutions of the Einstein-Maxwell-scalar theory that could describe an evolving charged black (white) hole or wormhole has been obtained in [17]. A magnetized rotating wormhole was obtained in [18] as an exact solution to the Einstein equations. The recent papers $[19,20]$ have presented specific examples of static traversable wormholes in Einstein-Dirac-Maxwell theory in four space-time dimensions, without explicit introduction of exotic matter, so that the Dirac fields themselves exhibit exotic properties [21]. Static, spherically symmetric wormhole solutions of asymptotically safe gravity have been recently studied in [22]. Wormholes were also considered in GR with Chaplygin gas [23], various models of phantom energy and quintessence, including models of matter with the energy-momentum tensor (EMT) structure of a perfect fluid [24-30]. In [24], it was shown that static, spherically symmetric wormholes with two flat or AdS asymptotic regions cannot exist in GR with any source matter with isotropic pressure, therefore, all such perfect-fluid wormholes contain their exotic sources only in a bounded region, surrounded by vacuum, with thin shells on the boundaries. In this connection, we should also mention a large class of wormhole models built completely using the thin-shell formalism, where the whole amount of exotic matter is concentrated on a thin shell; among them, Refs. [31,32] are probably the first examples.

Much of the literature on wormholes is devoted to studies of static, spherically symmetric configurations. Their important generalization is connected with dynamic solutions. Dynamic wormholes were considered in various aspects, and one of the ways of building these models $[28,33-37]$ is to add a time-dependent scaling factor to an otherwise static metric. Dynamic models of wormholes were also constructed using the thin shell formalism [38]. General properties of arbitrary dynamic wormholes are discussed in [39,40].

As mentioned above, a basic problem of wormhole physics is the necessity of exotic matter for their existence, at least in the case of static configurations in GR [10,41] and a wide class of scalar-tensor and $f(R)$ theories of gravity [42]. Rotational degrees of freedom are apparently able to replace exotic matter at wormhole construction. At least, some examples of rotating cylindrically symmetric wormhole models in GR have been built without NEC violation [43-45]; the recent examples with a static metric $[19,20]$ contain a Dirac field involving spin; one can also mention exotic-free solutions in the Einstein-Cartan theory $[46,47]$ containing nonzero torsion.

Dynamic wormholes can also avoid NEC violation, at least in cases where a static earlytime or late-time asymptotic behavior is absent. Such wormhole models with cosmologicaltype metrics are known, for example, in GR with electromagnetic fields described by some special forms of nonlinear electrodynamics $[48,49]$.

In this paper, we study the possible existence of traversable wormholes in GR with another classical and nonexotic form of matter, widely used in various problems of astrophysics and cosmology, namely, dustlike matter, with or without an electromagnetic field. The general spherically symmetric solution to the Einstein equations with dustlike matter was obtained by Lemaître and Tolman in 1933-1934 [50,51] and studied by Bondi in 1947 [52]; in the literature it is called the Tolman or LTB solution. Its properties were further investigated, for example, in [53-55]. In the recent paper by Kashargin and Sushkov [56], collapsing dusty wormholes were constructed using a special choice of arbitrary functions in Tolman's solution.

Tolman's solution was extended to include electrically charged dust with a radial electromagnetic field in [57-62] (see also references therein), where the problem was completely solved under some special conditions and considered on the level of integrals of the Einstein-Maxwell equations in the general case. The full set of solutions for arbitrary charged distributions and arbitrary initial data were obtained by N.V. Pavlov [63] and studied in [64]; further extensions to plane and hyperbolic symmetries were considered in [65-67], see also references therein.

The present study is restricted to configurations with an external magnetic (or electric) fields and electrically neutral dust, so that if we obtain a wormhole, its every entrance 
will contain a "charge without charge" [3,4] due to a bundle of electric or magnetic lines of force threading the throat. Similar models with radial magnetic fields and a particular choice of initial data have been studied in $[68,69]$, while here we consider the conditions for wormhole existence under general initial data.

It is worth noting that wormhole throats are defined in different ways by different authors. In the static case, different definitions agree with each other whereas in dynamic models contradictions may arise. General definitions of a throat including time-dependent metrics are considered, for example, in [40,70,71]. Following the papers [33-35], we will use a definition that rests on the properties of the 3-geometry of spatial sections of a given space-time.

It turns out that wormhole throats in the solution under study can be located only in a T-region, i.e., a nonstatic region such as that inside a black hole. Therefore, solutions containing a throat can lead to wormholes as global entites only in a cosmological context, but if we try to inscribe such a solution to an asymptotically flat space-time, we can only obtain black holes which also possess some properties of interest.

The paper is organized as follows. In Section 2, we reproduce a derivation of the generalized Tolman solution describing the evolution of a spherically symmetric cloud of dustlike matter in the presence of an external electric or magnetic field, without an explicitly introduced source. In Section 3 we investigate the possible existence of throats and traversable wormholes. Section 4 describes some particular examples, and Section 5 is a conclusion.

\section{Tolman's Solution with an Electric or Magnetic Field}

Let us consider a generalization of Tolman's solution [50-52] (to be called the $q$-Tolman solution), describing the evolution of a spherically symmetric cloud of electrically neutral dustlike matter in the presence of an external electric or magnetic field. In what follows, for certainty, we prefer to speak of a magnetic field since it is more realistic from an astrophysical viewpoint, though all solution to be discussed can be interpreted as those with an electric field.

If we choose a comoving reference frame for neutral dust particles, it is also a geodesic reference frame for them, and the metric can be taken in the synchronous form

$$
d s^{2}=d \tau^{2}-\mathrm{e}^{2 \lambda(R, \tau)} d R^{2}-r^{2}(R, \tau) d \Omega^{2},
$$

where $\tau$ is the proper time along the particle trajectories labeled by different values of the radial coordinate $R, \lambda(R, \tau)$ and $r(R, \tau)$ are functions of $\tau$ and $R$.

The energy-momentum tensor (EMT) of dustlike matter in the comoving frame has the form $T_{\mu}^{v[d]}=\rho u_{\mu} u^{v}$, where $\left(u^{v}\right)=(1,0,0,0)$ is a velocity four-vector, and $\rho$ is the energy density. The only nonzero component of the EMT of dust is $T_{0}^{0[d]}=\rho$, while for the electromagnetic field the EMT may be presented as

$$
T_{\mu}^{v[\mathrm{em}]}=\frac{q^{2}}{8 \pi G r^{4}} \operatorname{diag}(1,1,-1,-1),
$$

where $q$ may be interpreted as an electric or magnetic charge in proper units [72,73].

The nontrivial components of the Einstein equations may be written in the form

$$
\begin{aligned}
& 2 r \ddot{r}+\dot{r}^{2}+1-e^{-2 \lambda} r^{\prime 2}=\frac{q^{2}}{r^{2}}, \\
& \frac{1}{r^{2}}\left(1+\dot{r}^{2}+2 r \dot{r} \dot{\lambda}\right)-\frac{\mathrm{e}^{-2 \lambda}}{r^{2}}\left(2 r r^{\prime \prime}+r^{\prime 2}-2 r r^{\prime} \lambda^{\prime}\right)=8 \pi G \rho+\frac{q^{2}}{r^{4}}, \\
& \dot{r}^{\prime}-\dot{\lambda} r^{\prime}=0 .
\end{aligned}
$$


Equation (5) is easily integrated in $\tau$ giving

$$
\mathrm{e}^{2 \lambda}=\frac{r^{\prime 2}}{1+f(R)},
$$

where $f(R)$ is an arbitrary function satisfying the condition $1+f(R)>0$. Substituting (6) into (3), we obtain the equation

$$
2 r \ddot{r}+\dot{r}^{2}=f(R)+\frac{q^{2}}{r^{2}}
$$

whose first integral is

$$
\dot{r}^{2}=f(R)+\frac{F(R)}{r}-\frac{q^{2}}{r^{2}} .
$$

This expression makes clear the physical meaning of the function $f(R)$ : since $\dot{r}$ can be loosely understood as the radial velocity of a dust particle, $f(R)$ specifies the initial dust velocity distribution: for $f \geq 0$ it is the particle velocity squared at large $r(f>0$ and $f=0$ correspond to hyperbolic and parabolic motion, respectively). If $f(R)<0$ (elliptic motion), the particle cannot reach infinity, and the condition $\dot{r}=0$ in (8) shows the maximum value of $r$ accessible to it.

The meaning of the other arbitrary function, $F(R)$, becomes clear if we substitute (6) and (8) into the constraint Equation (4). We obtain

$$
\rho=\frac{1}{8 \pi G} \frac{F^{\prime}(R)}{r^{2} r^{\prime}}
$$

or

$$
F(R)=8 \pi G \int \rho r^{2} r^{\prime} d R .
$$

Assuming that in the initial configuration (before collapse) there was a regular center, so that $F=0$ at $r=0$, we can write

$$
F(R)=2 G M(R), \quad M(R)=4 \pi \int_{0}^{r} \rho r^{2} r^{\prime} d R,
$$

so that $M(R)$ is the mass function equal to the mass of a spherical body including all matter inside the sphere of given radius $r$, and $F(R)$ is the corresponding Schwarzschild radius. If there is no regular center, such a direct interpretation becomes impossible. However, the relation $F(R)=2 G M(R)$ can also be used in cases without a regular center (or even if there is no center at all).

Equation (8) can be further integrated, and the solution depends on the sign of $f(R)$ :

$$
\begin{array}{ll}
f>0: & \pm\left[\tau-\tau_{0}(R)\right]=\frac{1}{f} \sqrt{f r^{2}+F r-q^{2}}-\frac{F}{2 f^{3 / 2}} \ln \left(F+2 f r+2 \sqrt{f} \sqrt{f r^{2}+F r-q^{2}}\right), \\
f=0: \quad \pm\left[\tau-\tau_{0}(R)\right]=\frac{2 \sqrt{F r-q^{2}}\left(F r+2 q^{2}\right)}{3 F^{2}}, \\
f<0: \quad \pm\left[\tau-\tau_{0}(R)\right]=\frac{1}{h} \sqrt{-h r^{2}+F r-q^{2}}+\frac{F}{2 h^{3 / 2}} \arcsin \frac{F-2 h r}{\sqrt{F^{2}-4 h q^{2}}}, \quad h(R):=-f(R) .
\end{array}
$$

Please note that the elliptic model (14) is admissible only in the case $F^{2}-4 h q^{2} \geqslant 0$. The new arbitrary function $\tau_{0}(R)$ corresponds to a choice of clock synchronization between different spheres with fixed $R$ (Lagrangian spheres).

To obtain a global solution including both a dust distribution and an external electrovacuum region described by the Reissner-Nordström metric, it is necessary to describe such an electrovacuum region in a reference frame suitable for smooth matching to the 
internal solution. This problem is easily solved since the external solution in a proper form is obtained from the presently discussed solution (to be called " $q$-Tolman solution") by putting $F(R)=$ const whence $\rho=0$. The resulting solution reproduces the ReissnerNordström [72,73] metric in a geodesic reference frame. A global configuration is then represented by the $q$-Tolman solution in which $F(R)$ is variable in a certain range of $R$, say, $R<R_{0}$, and constant at larger $R$, so that $F(R)=F\left(R_{0}\right)=2 G M\left(R_{0}\right), M\left(R_{0}\right)$ being the Schwarzschild mass.

This makes possible an interpretation of the arbitrary function $M(R)$ independent from any assumptions on a center of the configuration: at each $R$, it is the Schwarzschild mass of the external (electro)vacuum solution that can be joined to the internal one at this particular value of $R$.

\section{Possible Wormhole Throats}

As follows from (9), to keep the density positive, we must require $F^{\prime} / r^{\prime}>0$, but it is not necessary to assume that both $F^{\prime}$ and $r^{\prime}$ are positive. This means that one cannot exclude the existence of regular minimum or maximum values of $r$ (at given $\tau$ ), which can be interpreted as throats and equators, respectively.

As already said in the Introduction, there are different definitions of a wormhole throat suitable for general time-dependent metrics. We will use the definition according to [33-35]. Therefore, let us call a throat a regular minimum of the spherical radius $r(R, \tau)$ at given $\tau$ (that is, in a fixed spatial section of our space-time), and let us try to understand whether it is possible to build a wormhole configuration based on the $q$-Tolman solution. The first thing to do is to find out the conditions to be satisfied on a wormhole throat.

Considering a section $\tau=$ const, we can write the 3D spatial metric as

$$
d l_{(3)}^{2}=\frac{r^{\prime 2} d R^{2}}{1+f(R)}+r^{2}(R) d \Omega^{2} .
$$

where $r(R)=\left.r(R, \tau)\right|_{\tau=\text { const. }}$.

Using the freedom to choose any radial coordinate $R$, we might simply take (at fixed $\tau) R=r$, but then, if $r$ has a minimum, it is not an admissible coordinate precisely at this minimum, the throat. To avoid using such undesirable radial coordinates, we can take the manifestly admissible Gaussian coordinate $l$, such that $d l=\left|g_{R R}\right|^{1 / 2} d R$ is the element of length in the radial direction. Then the throat conditions are

$$
\frac{d r}{d l}=0, \quad \frac{d^{2} r}{d l^{2}}>0
$$

(assuming a generic minimum and ignoring possible high-order ones, with a zero secondorder derivative). The first condition implies that at the throat, $R=R_{\text {th }}$,

$$
\frac{d r}{d l}=\sqrt{1+f\left(R_{\mathrm{th}}\right)}=0 \Rightarrow f\left(R_{\mathrm{th}}\right)=-1 \text { or } h\left(R_{\mathrm{th}}\right)=1 .
$$

We immediately obtain that only elliptic models (14) can be suitable for wormhole construction. Furthermore, since in general, to keep the metric (1) nondegenerate, we must have $1+f=1-h>0$, and $h=1$ may occur only at a particular value of $R$, it is clear that $R=R_{\text {th }}$ must be a maximum of $h(R)$, and it should be $h^{\prime}\left(R_{\mathrm{th}}\right)=0$ and $h^{\prime \prime}\left(R_{\mathrm{th}}\right)<0$. The second condition (16) leads to

$$
\left.\frac{d^{2} r}{d l^{2}}\right|_{R=R_{\mathrm{th}}}=-\left.\frac{h^{\prime}}{2 r^{\prime}}\right|_{R=R_{\mathrm{th}}}>0 .
$$

Thus, $r^{\prime}$ must vanish at $R=R_{\text {th }}$ along with $h^{\prime}$. A finite limit of $h^{\prime} / r^{\prime}$ may be found using the L'Hospital rule. The conditions (17) and (18) impose restrictions on the functions $F(R)$ and $h(R)$. 
A special case of functions $F(R)$ and $f(R)$ satisfying the throat conditions (17) and (18) was considered in [56]. In this paper, we consider the general case without specifying the functions $F(R)$ and $f(R)$ and test the traversability of the models. The point is that the existence of a throat is only a necessary condition for having a wormhole: it is also necessary to require the absence of horizons which are only one-way traversable, and even without horizons the traversability may be destroyed by the time evolution.

For the elliptic models (14) it is convenient to introduce the parametric representation,

$$
\begin{aligned}
r & =\frac{F}{2 h}(1-\Delta \cos \eta), \\
\pm\left[\tau-\tau_{0}\right] & =\frac{F}{2 h^{3 / 2}}(\eta-\Delta \sin \eta), \quad \Delta=\sqrt{1-\frac{4 h q^{2}}{F^{2}}}
\end{aligned}
$$

where $0<\Delta \leq 1$, and $\Delta=1$ corresponds to Tolman's solution without an electromagnetic field. We see that at $q \neq 0$, hence $\Delta<1$, the model is free from singularities characterized by $r=0$. On the other hand, in the special case $\Delta=1(q=0)$, the solution reduces to Friedmann's closed isotropic model filled with dust [53] under the assumptions

$$
F(\chi)=2 a_{0} \sin ^{3} \chi, \quad h(\chi)=\sin ^{2} \chi, \quad a_{0}=\mathrm{const}
$$

(the radial coordinate $R=\chi$ is here a "radial angle" of a 3D sphere), and we have

$$
r=r(\eta, \chi)=a(\eta) \sin \chi, \quad a(\eta)=a_{0}(1-\cos \eta),
$$

$a(\eta)$ being the cosmological scale factor.

An important question for our wormhole study is the allocation of $\mathrm{R}$ - and T-regions separated by apparent horizons [74]. The answer follows from the definition of R-and T-regions, according to which a T-region is the one where the gradient of $r(R, \tau)$ is timelike, an R-region where it is spacelike, and it is null at a horizon. Evidently,

$$
r^{\prime \alpha} r_{, \alpha}=\dot{r}^{2}-\mathrm{e}^{-2 \lambda} r^{\prime 2}=-1+\frac{F(R)}{r}-\frac{q^{2}}{r^{2}},
$$

where the second equality sign follows from (6) and (8). Recalling that $F(R)=2 G M(r)$, we observe that the R- and T-regions occur in full similarity with the Reissner-Nordström space-time, where, instead of the mass of a central body, there stands the current value of the mass function $M(R)$.

Recalling that a possible wormhole throat can only occur at $h=1$ and substituting the condition (17) and $r(R, \tau)$ from (19), we finally obtain that at such a throat

$$
\left.r^{\prime \alpha} r_{, \alpha}\right|_{R_{\mathrm{th}}}=\frac{1}{r^{2}} \sin ^{2} \eta\left(\frac{F^{2}}{4}-q^{2}\right) \geq 0 .
$$

It means that a seeming wormhole throat is located, in general, in a T-region, and only at $\eta=\pi n, n \in \mathbb{N}$ (at which the radius $r$ for given $R$ takes extremal values as a function of $\tau)$, the throat coincides with a horizon.

This means that a would-be asymptotically flat wormhole configuration that could be constructed from the $q$-Tolman solution cannot be traversable and, if matched with an external Reissner-Nordström solution at some value of $R$, actually represents a black hole. This also applies to the special case $q=0$, the absence of a magnetic field, such that we return to the Tolman solution: as follows from (23), this solution also does not admit traversable wormholes with flat asymptotic regions. If it is matched to an external Schwarzschild solution at some value of $R$, we obtain a black hole model with a dust source. In particular, this applies to the seemingly wormhole model considered in [56].

It may seem that such configurations with throats can be used to construct regular black hole configurations. We will see, however, that even though $r>0$ for all Lagrangian spheres if $q \neq 0$, all solutions with a throat exist in a finite time interval, beginning and 
ending with shell-sticking singularities due to $r^{\prime}=0$. Another point of interest can be the existence of dynamic cosmological wormholes, which can be obtained if we do not match the $q$-Tolman solution to a Reissner-Nordström one on both sides of the throat but, instead, require Freidmann-Robertson-Walker asymptotics on both sides, see Section 4 .

One more observation is that, as already follows from Equation (8), the elliptic mode of dust motion is only compatible with charges $q<F / 2=G M$, corresponding to the black hole branch of the Reissner-Nordström solution. Larger charges, which could seem to favor the existence of wormholes, require the hyperbolic mode, which is in turn in conflict with the condition (17).

As follows from the expression (9) for the dust density, $\rho \rightarrow \infty$ if either $r \rightarrow 0$ or $r^{\prime} \rightarrow 0$, except for the case where both $r^{\prime} \rightarrow 0$ and $F^{\prime} \rightarrow 0$ at finite $r$, such that $F^{\prime} / r^{\prime}$ remains finite, precisely the situation of interest for us at a wormhole throat. The absence of a singularity under these conditions is confirmed by the expression for the Kretschman scalar $\mathcal{K}^{1}$

$$
\mathcal{K}(R, t)=3 \frac{F^{\prime 2}}{r^{\prime 2} r^{4}}-8 \frac{F^{\prime} F}{r^{\prime} r^{5}}+12 \frac{F^{2}}{r^{6}}+20 \frac{F^{\prime} q^{2}}{r^{\prime} r^{6}}-48 \frac{F q^{2}}{r^{7}}+56 \frac{q^{4}}{r^{8}} .
$$

We have seen that possible throats require simultaneous vanishing of the three derivatives $r^{\prime}, F^{\prime}$ and $h^{\prime}$, with finite limits of the ratios $F^{\prime} / r^{\prime}$ (see (9) and (24)) and $h^{\prime} / r^{\prime}$ (see (18)), and we also try to keep the density $\rho$ positive.

By (19), the derivative $r^{\prime}$ on a constant- $\tau$ spatial section of our space-time is given by

$$
\begin{aligned}
& r^{\prime}=\frac{F h^{\prime} N_{1}(R, \eta)+2 h F^{\prime} N_{2}(R, \eta)}{4 \Delta h^{2}(1-\Delta \cos \eta)} \\
& N_{1}(R, \eta)=\cos \eta-3 \Delta+3 \Delta^{2}(\eta \sin \eta+\cos \eta)+\Delta^{3}\left(-2+\cos ^{2} \eta\right) \\
& N_{2}(R, \eta)=-\cos \eta+2 \Delta-\Delta^{2}(\cos \eta+\eta \sin \eta) .
\end{aligned}
$$

Since at a throat $R=R_{\mathrm{th}}$ the quantities $F^{\prime} / r^{\prime}$ and $h^{\prime} / r^{\prime}$ are finite and nonzero (though have different signs), $r^{\prime}, h^{\prime}$ and $F^{\prime}$ are infinitesimal quantities of the same order of magnitude near such a throat.

We can summarize that on a throat $R=R_{\text {th }}$ we must have

$$
\begin{array}{ll}
h=1, & h^{\prime}=0, \quad h^{\prime \prime}<0, \\
F^{\prime}=0, \quad r^{\prime}=0, & \frac{h^{\prime}}{r^{\prime}}<0, \quad \frac{F^{\prime}}{r^{\prime}}>0 .
\end{array}
$$

Furthermore, we have everywhere $F^{2}-4 h q^{2}>0$ and $\Delta \leq 1$.

For a further analysis, consider the $\operatorname{limit}_{R \rightarrow R_{\mathrm{th}}} \frac{F h^{\prime}}{F^{\prime} h}=-B$ with $B=$ const $\geq 0$. Then we have on the throat

$$
\left.r^{\prime}\right|_{R_{\mathrm{th}}}=\frac{F^{\prime}\left(2 N_{2}-B N_{1}\right)}{4 \Delta(1-\Delta \cos \eta)}
$$

and it vanishes only where $F^{\prime}=0$ or $N_{*}=2 N_{2}-B N_{1}=0$. The density (9) and the second-order derivative $d^{2} r / d l^{2}(18)$ have there the values

$$
\begin{aligned}
& \rho\left(R_{\mathrm{th}}, \eta\right)=\left.\frac{\Delta(1-\Delta \cos \eta)}{2 \pi G r^{2}\left(2 N_{2}-B N_{1}\right)}\right|_{R_{\mathrm{th}}}, \\
& \left.\frac{d^{2} r}{d l^{2}}\right|_{R_{\mathrm{th}}}=\left.\frac{2 B}{F} \frac{\Delta(1-\Delta \cos \eta)}{2 N_{2}-B N_{1}}\right|_{R_{\mathrm{th}}} .
\end{aligned}
$$


These quantities blow up when $N_{*}=2 N_{2}-B N_{1}=0$, while all other factors are strictly positive ( $F>0$ by assumption). However, the quantity $N_{*}$ as a function of $\eta$ has, in general, an alternating sign, as follows from the expressions (see also Figure 1).

$$
\begin{aligned}
& \left.N_{*}\right|_{\eta=0,2 \pi}=-(1-\Delta)^{2}[2+B(1-\Delta)] \leq 0, \\
& \left.N_{*}\right|_{\eta=\pi}=(1+\Delta)^{2}[2+B(1+\Delta)]>0 .
\end{aligned}
$$

Therefore we inevitably have $N_{*}=0$, hence a singularity, at (at least) two values of $\eta$, say, $\eta_{1}$ and $\eta_{2}$, in the range $\eta \in(0,2 \pi)$ for any $\Delta<1(q \neq 0)$. In the case $q=0, \Delta=1$ (pure dust), $N_{*}$ vanishes at $\eta_{1,2}=0,2 \pi$ and is positive at $\eta \in(0,2 \pi)$. The quantity $N_{*}$ is plotted in Figure 1 for some values of $B$ and fixed values of $\Delta$. Plots of the density $\rho$ will be shown below for a specific example.
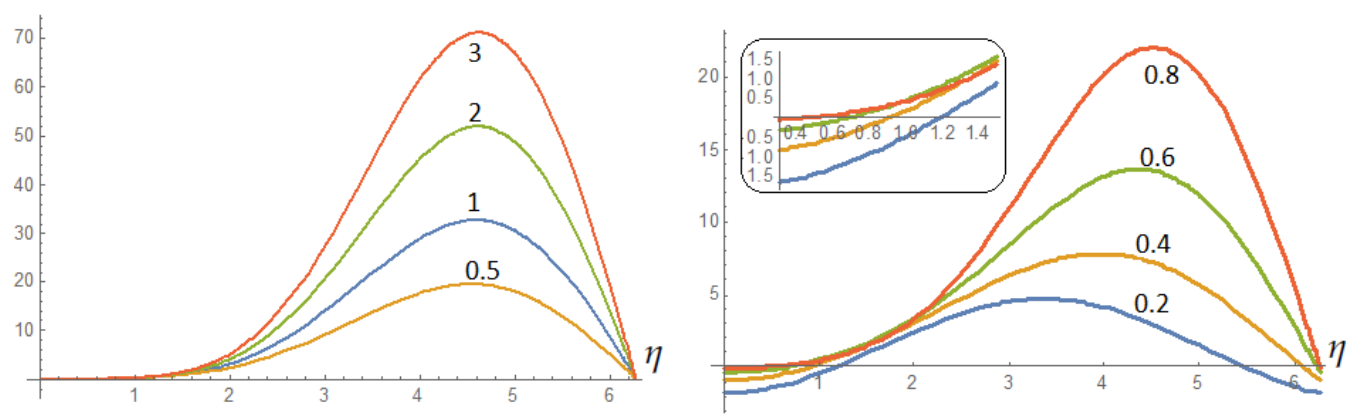

Figure 1. Time dependence of $N_{*}=2 N_{2}-B N_{1}$. Left: $\Delta=1, B=0.5,1,2,3$; right: $B=1, \Delta=$ $0.2,0.4,0.6,0.8$; for other values $B$ and $\Delta$ the plots looks similarly. The inset on the right panel clarifies the points where $N_{*}=0$. Please note that $\rho$ and $d^{2} r / d l^{2}$ at $R=R_{\text {th }}$ are positive or negative simultaneously with $N_{*}$.

We conclude that a nonsingular evolution of a throat $R=R_{\text {th }}$ with positive density $\rho$ can take place at times $\eta_{1}<\eta<\eta_{2}$ at which $N_{*}>0$. For other Lagrangian spheres $R=$ const similar time limits will be different due to $R$ dependence of $F$ and $h$.

\section{Some Particular Models}

\subsection{A Special Wormhole Solution}

Let us try to construct an example of a $q$-Tolman wormhole solution, choosing the following simple functions of $R$ in agreement with the requirements (26):

$$
h(R)=\frac{1}{1+R^{2}}, \quad f(R)=2 b\left(1+R^{2}\right) \Rightarrow \Delta=\sqrt{1-\frac{q^{2}}{b^{2}\left(1+R^{2}\right)^{3}}},
$$

( $b=$ const $>0$, specifying a length scale), so that

$$
r(R, \eta)=b\left(1+R^{2}\right)^{2}(1-\Delta \cos \eta), \quad r^{\prime}(R, \eta)=\frac{b R\left(1+R^{2}\right)\left(2 N_{2}-N_{1}\right)}{\Delta(1-\Delta \cos \eta)}
$$

with $N_{1,2}$ defined in Equaiton (25). The throat is located at $R=0$, and the whole solution is symmetric with respect to it. The density $\rho(9)$ and the quantity $d^{2} r / d l^{2}$ at $R=0$ then read

$$
\begin{aligned}
\rho(R, \eta) & =\frac{\Delta}{2 \pi G b^{2}\left(1+R^{2}\right)^{5}(1-\Delta \cos \eta)\left(2 N_{2}-N_{1}\right)}, \\
\left.\frac{d^{2} r}{d l^{2}}\right|_{R=0} & =\left.\frac{\Delta(1-\Delta \cos \eta)}{b\left(2 N_{2}-N_{1}\right)}\right|_{R=0} .
\end{aligned}
$$


As already noted, different signs of the derivatives of $h(R)$ and $f(R)$, under the condition $2 N_{2}(R, \eta)-N_{1}(R, \eta)>0$, provide the validity of the throat conditions (26) at $R=0$ and, by continuity, in some its neighborhood, but the same is not guaranteed at all $R$ and $\eta$.

The time dependence of the throat radius is shown in Figure 2, and the density $\rho$ on the throat in Figure 3 for different values of $q$, where dashed lines show the asymptotes of the function. Finite positive density values are observed for a limited period of time $\eta \in\left(\eta_{1}, \eta_{2}\right)$ while $2 N_{2}-N_{1}>0$, between two singularities where $\rho$ and $\mathcal{K}$ diverge. Outside this interval, in the case $q \neq 0$, the density changes its sign along with $d^{2} r / d l^{2}$, therefore the throat conditions hold together with the condition $\rho>0$.

Thus, we observe a good wormhole behavior of our solution at the time interval $\eta \in\left(\eta_{1}, \eta_{2}\right)$. With decreasing charge, this interval increases; and at $q=0$ we have $\eta_{1}=0$, $\eta_{2}=2 \pi$. Outside the throat (at $R \neq 0$ ), the plots look similarly, but the singularities occur at other time instants.
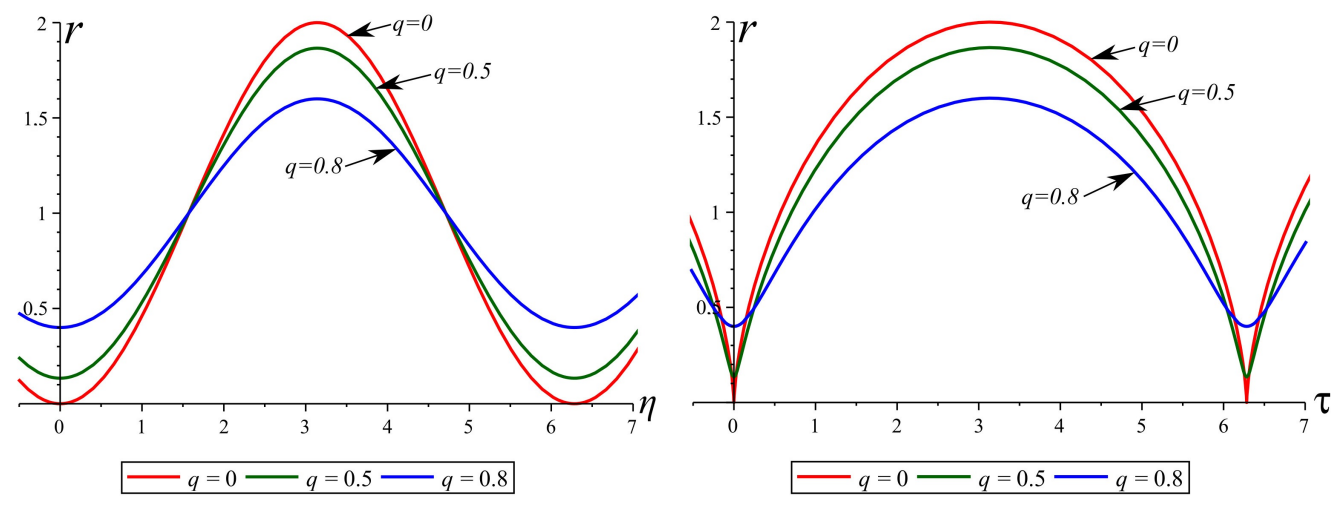

Figure 2. Time dependence of the throat radius for $q=0,0.5,0.8$ in terms of $\eta$ (left) and in terms of $\tau$ (right).

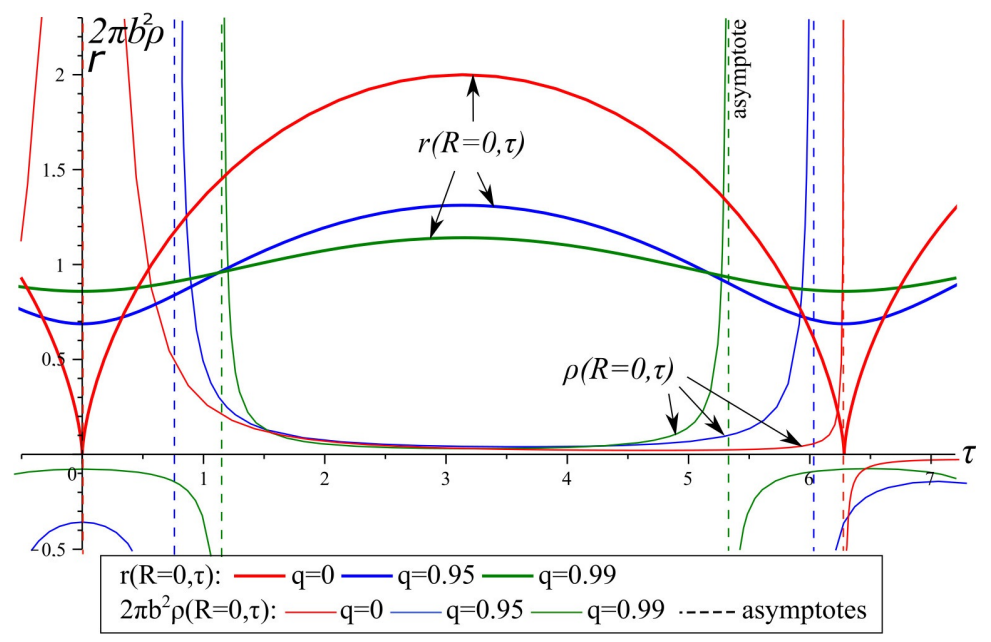

Figure 3. Time dependence of the functions $2 \pi b^{2} \rho$ (thin lines) and $r(0, \tau)$ (thick lines) on the throat $R=0$ for $q=0,0.95,0.99$ in the model (31). Dashed lines show the asymptotes. For other values of $q$ the plots look in a similar way.

\subsection{Wormholes in a Dust-Filled Universe}

Now, let us look how such a wormhole solution can be inscribed into the closed isotropic cosmological model characterized by the relations (20) and (21). To that end we note that to match two $q$-Tolman space-times, characterized by different functions $F(R)$ and $h(R)$, at some value of the radial coordinate $R=R^{*}$, one should above all identify the hypersurface $\Sigma\left(R=R^{*}\right)$ as is viewed from different sides since in unified space-time it 
should be a single hypersurface. This means that the metric tensor should be continuous on $\Sigma$. With the metric (1) it means simply $\left[r^{2}(R, \tau)\right]=0$ (where the square brackets, as usual, denote jumps at a transition across $\Sigma$ ), while by (1) $g_{\tau \tau} \equiv 1$ on both sides.

Next, according to [75,76], discontinuities in the second quadratic form of $\Sigma$ correspond to a nonzero stress-energy tensor of the surface matter content, and to avoid its presence we should require that it should have no jumps. For the metric (1), this requirement leads to $\left[\mathrm{e}^{-\lambda} g_{\tau \tau}^{\prime}\right]=0$ (which trivially holds) and $\left[\mathrm{e}^{-\lambda} r^{\prime}\right]=0$. Taking into account (6) and (19), we obtain

$$
[r]=0, \quad\left[\mathrm{e}^{-\lambda} r^{\prime}\right]=0 \Rightarrow[h]=0, \quad[F]=0, \quad[q]=0 .
$$

Thus, to match two $q$-Tolman solutions on a surface $\Sigma\left(R=R^{*}\right)$, we must identify the values of $F\left(R^{*}\right)$ and $h\left(R^{*}\right)$ as well as the parameter $q$ in these solutions. Then, by virtue of (19), these matching conditions are valid at all times at which both solutions remain regular. Furthermore, there is no necessity to adjust the choice of the radial coordinates on different sides of $\Sigma$ because both quantities $r$ and $\mathrm{e}^{-\lambda} r^{\prime}$ are insensitive to reparametrizations of $R$, and the same is true for the functions $h(R)$ and $F(R)$ which behave as scalars at such reparametrizations.

Now, let us apply the conditions (35) to the solution (20) and (21) with $q=0$ and (31) and (32) on the other, also putting $q=0$, and specifying the junction surface by some values $\chi=\chi^{*}$ and $R=R^{*}$. We then obtain

$$
R^{*}=\cot \chi^{*}, \quad b=a_{0} \sin ^{5} \chi^{*} .
$$

that provides matching at $R^{*}>0$. Since the functions involved in (31) are even, the same kind of matching can be implemented at $R^{*}<0$. The whole composite model thus consists of two evolving closed Friedmann universes filled with dust and connected through a wormhole, forming a dumbbell-like configuration.

Some numerical estimates are in order. Assuming $a_{0} \sim 10^{28} \mathrm{~cm}$, approximately the size of the visible part of the Universe, let us require, on the other hand, that the throat radius $r_{\text {th }} \sim b$ should substantially exceed the Planck length, $b \gg 10^{-33} \mathrm{~cm}$. Then, using (36), we easily obtain

$$
\sin \chi^{*} \gg 10^{-12}, \quad R^{*} \ll 10^{12},
$$

which implies $r^{*}=r\left(R^{*}\right) \gg 10^{16} \mathrm{~cm} \sim 0.01$ light year. It means that the wormhole region should be rather large on the astronomic scale. If we assume $r^{*} \sim 100$ light years, a stellar cluster size, it follows $\chi \sim 10^{-8}$ and $r_{\text {th }} \sim 10^{20} l_{\mathrm{pl}} \sim 10^{-13} \mathrm{~cm}$, the throat having the size of an atomic nucleus. If the wormhole region is as large as a galaxy, $r^{*} \sim 30 \mathrm{kpc} \sim 10^{23} \mathrm{~cm}$, we obtain $\chi \sim 10^{-5}$, resulting in $r^{*} \sim 10^{3} \mathrm{~cm}$, a throat of macroscopic size.

The density Equation (9) for our model (31) with $q=0$ is given by

$$
\rho=\frac{1}{2 \pi G b^{2}} \frac{1}{\left(1+R^{2}\right)^{5}(1-\cos \eta)\left(2 N_{2}-N_{1}\right)}
$$

where $2 N_{2}-N_{1}$ is, for $\Delta=1$, a function of $\eta$, positive in the whole range $\eta \in(0,2 \pi)$. The time-dependent factor in (38) can be approximated by $1 / 10$, which is really true in a larger part of the time interval, and then, by order of magnitude, we have

$$
\rho \sim \frac{10^{-30}}{\left(1+R^{2}\right)^{5} \sin ^{10} \chi^{*}} \frac{\mathrm{g}}{\mathrm{cm}^{3}} .
$$

On the throat $R=0$ this gives large values: about $10^{50} \mathrm{~g} / \mathrm{cm}^{3}$ if $\chi^{*}=10^{-8}$ and about $10^{20} \mathrm{~g} / \mathrm{cm}^{3}$ if $\chi^{*}=10^{-5}$, much more than the nuclear density. On the other hand, on the junction surface $R=R^{*}$, since $R^{*} \sim 1 / \chi^{*}$, the density turns out to have a universal value independently from $\chi^{*}, \rho \sim 10^{-30} \mathrm{~g} / \mathrm{cm}^{3}$, of the order of mean cosmological density. 


\subsection{A Wormhole-like Structure inside a Schwarzschild or Reissner-Nordström Black Hole}

Let us now discuss the dynamics of our model with a throat assuming that dust matter is distributed in some spherical layer containing a throat, and $R= \pm R^{*}$ is the radial coordinate of the outer layer (Lagrangian sphere) on which the $q$-Tolman solution is matched to the external Reissner-Nordström solution (or Schwarzschild in the case $q=0$ ). Thus, the external metric is

$$
d s^{2}=A(r) d t^{2}-\frac{d r^{2}}{A(r)}-r^{2} d \Omega^{2}, \quad A(r)=1-\frac{2 M}{r}+\frac{Q^{2}}{r^{2}},
$$

where $M$ and $Q$ are the mass and charge of the source (using units in which $G=1$ ), and we should consider the case $M>|Q|$ when the Reissner-Nordström space-time contains an event horizon with $r=r_{+}=M+\sqrt{M^{2}-Q^{2}}$ and a Cauchy horizons with $r=r_{-}=M-\sqrt{M^{2}-Q^{2}}$. Naturally, there must be such an outer surface on each side from the throat, with maybe different masses $M$ in the external regions but with the same charge $Q$. We can also construct a model with a closed universe, as described above, on one side from the throat and empty asymptotically flat space on the other, but here we do not consider such models.

As already discussed in Section 2, the Reissner-Nordström solution is obtained from the $q$-Tolman solution as its special case such that $F(R)=2 M=$ const and $q=Q$. According to (35), we must also have the jump $[h(R)]=0$ on the junction surfaces. Apart from this junction condition, the function $h(R)$ remains arbitrary but can be given a desired form by accordingly choosing the radial coordinate $R$, and with any such choice we are remaining in the geodesic reference frame in Reissner-Nordström space-time.

Consider the motion of the outer surface of this thick dust layer from the initial singularity to the final one. As follows from the examples we considered, both singularities for any Lagrangian surface in models under consideration (that is, containing throats) are located in a T-region, where the gradient of $r(R, \tau)$ is timelike. It means, in particular, that dust particles belonging to an outer surface are initially located inside a white hole and move in the direction of growing $r$. Further on they reach a maximum of $r$, and it is easy to prove that such a maximum occurs in the R-region, hence outside the event horizon $r=r_{+}$. Indeed, recall the integral (8) of the equations of motion and assume there the extremum condition $\dot{r}=0$, to obtain for the surface $R=R_{s}$

$$
h(R)=\frac{2 M(R)}{r}-\frac{q^{2}}{r^{2}}=1-A(r) \Rightarrow A\left(r_{\max }\right)=1-h(R)
$$

because the surface belongs to both internal and external regions. Since $h(R)<1$ for all $R$ except the throat, we obtain $A\left(r_{\max }\right)>0$, i.e., an R-region.

After reaching $r_{\max }$, the surface layer shrinks again towards the event horizon $r=r_{+}$, thus entering a black hole. The whole process takes a finite proper time $\tau$ of the surface particles, but from the viewpoint of a distant observer, the "star" of dust emerges from the horizon in the infinitely remote past and collapses back in the infinitely remote future.

All that happens in the same way irrespective of whether or not there is a wormholelike structure inside the horizon. If this structure is present, it means that there is another external Reissner-Nordström or Schwarzschild space-time on the other side of the throat. One more specific feature of models with a throat is that with $q \neq 0$ the whole evolution of all Lagrangian spheres lasts a finite proper time interval between two singularities at which $r^{\prime} \rightarrow 0$, the so-called shell-crossing, or shell-sticking singularities, while in the general case of $q$-Tolman solution, completely regular oscillating configurations are possible (recall that with $q \neq 0$ the electromagnetic field prevents reaching a singularity $r=0$ at which a Lagrangian sphere shrinks to a point, see, e.g., Figure 3 that illustrates the model evolution for a particular choice of the parameters in Equation (31)). 
An important feature of models with a throat is that the Cauchy horizon $r=r_{-}$is never reached by the outer surface since the throat is a minimum of $r$ at each $\tau$ and is located in a T-region between $r_{-}$and $r_{+}$.

A question of interest concerns the evolution of a throat $R=R_{\mathrm{th}}$. We know that the throat is located in a T-region while a maximum of $r(R, \tau)$ is reached in an R-region for all $R \neq R_{\mathrm{th}}$. How can it happen that the throat radius $r_{\mathrm{th}}=r\left(R_{\mathrm{th}}, \tau\right)$ has a maximum? The answer is that a maximum of $r_{\text {th }}$ is reached precisely at the horizon, a boundary between the R- and T-regions, and it is possible because $h\left(R_{\mathrm{th}}\right)=1$, so the reasoning used about the surface layer (see Equation (41)) does not work. In terms of the parametrization (19), we have a maximum of $r_{\text {th }}$ at $\eta=\pi$, when $r^{\alpha} r_{, \alpha}=0$.

\subsection{Photon Motion across the Dust Layer}

Now we would like to consider the radial motion of light in the model (31) of a dust layer, assuming that it is bounded by $|R|<R^{*}$ and is located between two copies of a Reissner-Nordström or Schwarzschild (if $q=0$ ) space-time. It is clear that a photon radially falling to such a black hole reaches the throat and has no other way than to travel further in the direction of another universe or maybe a distant part of the same universe. The question is whether or not it will go out from the dust layer in this "other" universe before the whole configuration reaches its final singularity. In other words, is the wormhole (or the wormhole part of space-time) traversable.

The radial null geodesic equation has the form following from the condition $d s=0$ applied to the metric (1),

$$
\frac{d R}{d \tau}= \pm \frac{\sqrt{1-h}}{r^{\prime}}= \pm \frac{(\operatorname{sign} R) \Delta(1-\Delta \cos \eta)}{\left(1+R^{2}\right)^{3 / 2}\left(2 N_{2}-N_{1}\right)},
$$

where we assumed $b=1$, and the minus and plus signs correspond to ingoing and outgoing light rays, respectively, in the region $R>0$. Of interest is the time interval where $2 N_{2}-N_{1}>0$, in which the right-hand side of (5) is finite and nonzero, therefore, in particular, the photon reaches the throat at finite proper time. By symmetry of the model $(R \leftrightarrow-R)$, it is sufficient to consider the motion from positive to negative values of $R$, choosing the minus sign in the region $R>0$ (the ingoing direction) and the plus sign for $R<0$, which thus cancels the function sign $R$. Thus, we obtain

$$
\frac{d R}{d \tau}=-\frac{\Delta(1-\Delta \cos \eta)}{\left(1+R^{2}\right)^{3 / 2}\left(2 N_{2}-N_{1}\right)} .
$$

Let us calculate the time derivative of the spherical radius $r(\tau, R(\tau))$ along the light ray $R=R(\tau)$ :

$$
\frac{d r(\tau, R(\tau))}{d \tau}=\frac{\partial r}{\partial \tau}+\frac{\partial r}{\partial R} \frac{d R}{d \tau}= \pm \sqrt{-h+\frac{F}{r}-\frac{q^{2}}{r^{2}}} \pm \sqrt{1-h},
$$

where $R=R(\tau)$ describes the radial motion of a photon according to (5), the first \pm sign corresponds to expansion (plus, at $\eta \in(0, \pi)$ ) or contraction (minus, at $\eta \in(\pi, 2 \pi)$ ) of the dust matter, while the second \pm sign corresponds to an ingoing (minus) or outgoing (plus) photon, i.e., growing or falling values of $R$ along the path. Please note that when a photon reaches the throat $\left(h\left(R_{\mathrm{th}}\right)=1\right)$, it moves tangentially to the paths of dust particles: $\partial r(\tau, R(\tau)) / \partial \tau \equiv \partial r / \partial \tau$.

Curiously, it can happen that the quantity $r(R, \tau)$ for a radially moving photon, as a function of time, can have an extremum even though $\partial R / \partial \tau$ has a constant sign (a photon crosses the Lagrangian spheres in a definite direction). From Equation (44) it follows that such an extremum of $r$, at which $d r(\tau, R(\tau)) / d \tau=0$, can take place only if the \pm signs before the two terms are opposite, which means that an extremum of $r$ is only possible at 
the contraction stage $(\eta>\pi)$ for an outgoing photon or at the expansion stage $(\eta<\pi)$ for an ingoing photon. Specifically, we have $d r(\tau, R(\tau)) / d \tau=0$ at a surface where

$$
r^{2}-F r+q^{2}=0,
$$

that is, at the apparent horizon inside the dust layer, a surface where $r^{, \alpha} r_{, \alpha}=0$ in the metric (1).

Equation (44) is the equation of radial motion of a photon in the dust layer, and for our example (31) it gives

$$
\frac{d r(\tau, R(\tau))}{d \tau}=\frac{1}{\sqrt{1+R^{2}}}\left(\frac{\Delta \sin \eta}{1-\Delta \cos \eta}-R\right)
$$

where $\eta$ is meant as a function of $\tau$ and $R$, determined by the relations

$$
r=\left(1+R^{2}\right)^{2}(1-\Delta \cos \eta), \quad \tau=\left(1+R^{2}\right)^{5 / 2}(\eta-\Delta \sin \eta) .
$$

We consider photons (or light rays) that move radially in the direction from the region $R>0$ to the region $R<0$, which corresponds to the minus sign in the second term in (44). For motion in the opposite direction, one should replace $R \rightarrow-R$ in Equation (46). In the model with $q \neq 0$, the quantity $d r / d \tau$ is always finite; while in the model with pure dust $(q=0)$ it diverges at $\eta=0$ and $\eta=2 \pi$; in all cases, a photon crosses the vicinity of the throat $R=0$ in a finite proper time. The results of numerical integration of Equation (46) in the cases $q=0$ and $q \neq 0$ are shown in Figures 4 and 5 .

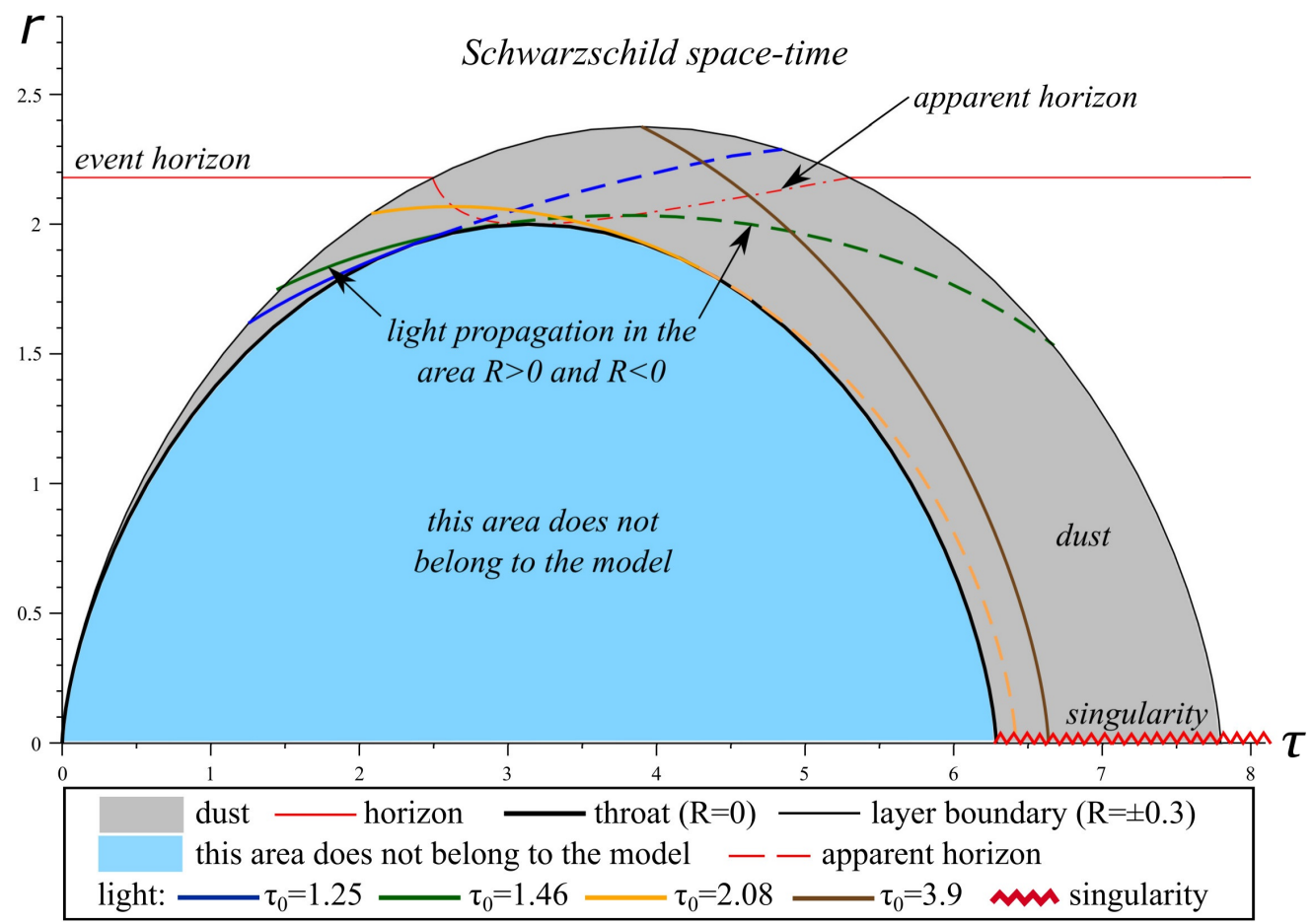

Figure 4. The figure shows: (1) The time dependence $r(\tau, R)$ for shells (Lagrangian spheres) $R=$ const in the dust layer (gray area), the throat $R=0$ and the outer surface $R= \pm 0.3$ in the case $q=0$. (2) The time dependence $r(\tau)$ of ingoing radial photons coming from the outer shell $R=0.3$ towards the throat $R=0$ at different times $\tau_{0}$; reaching the throat, a photon path passes from the region $R>0$ (solid line) to the region $R<0$ (dashed line). (3) The singularity, presented by a broken red line. (4) The event horizon in vacuum and the apparent horizon inside the dust layer, presented by solid and dashed red lines, respectively. 


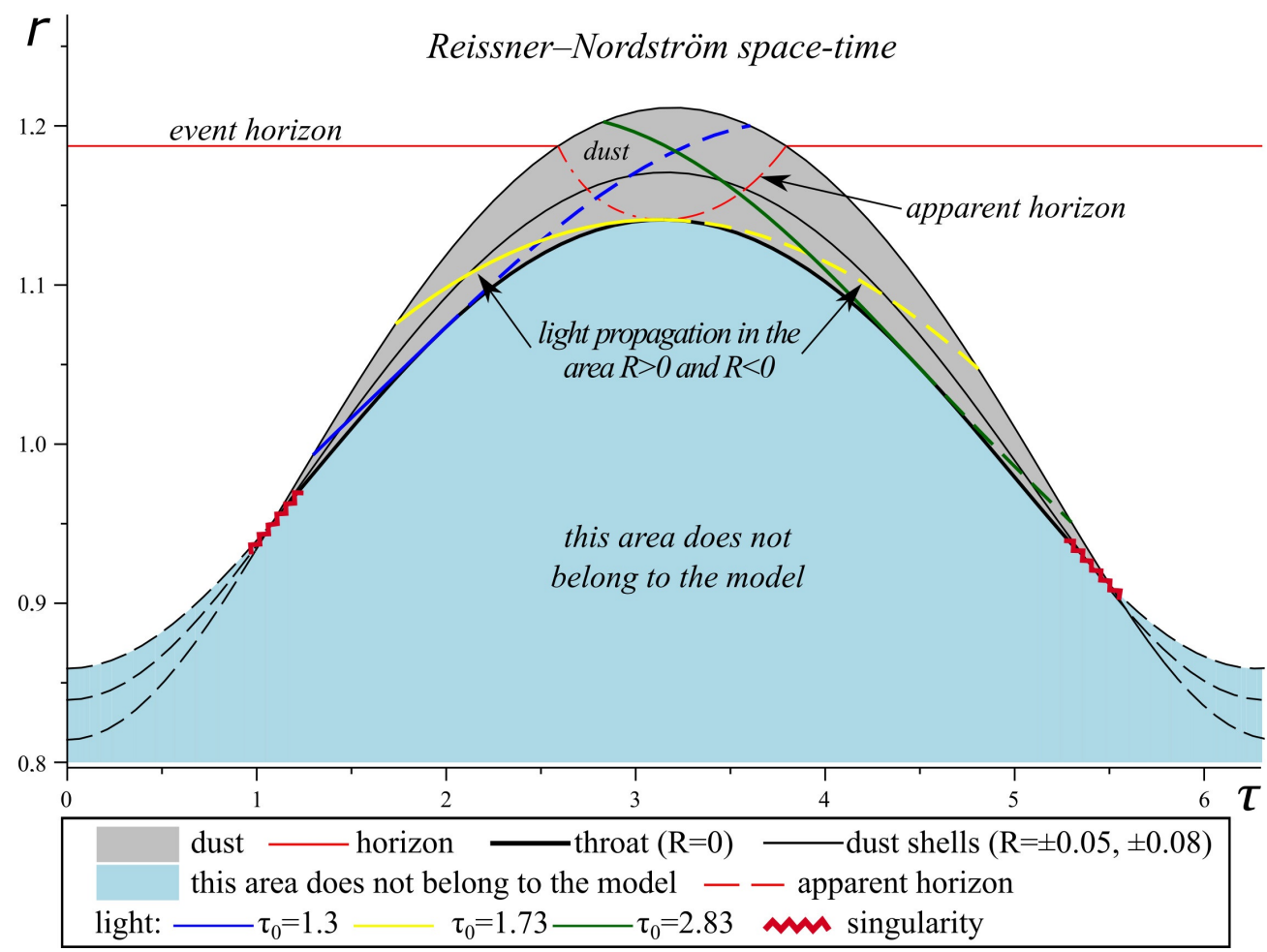

Figure 5. The figure shows the time dependence $r(R, \tau)$ of dust shells $\left(R_{0}=0, \pm 0.05, \pm 0.08\right.$, $|R| \leq 0.8)$ in the case $q=0.99$. The curve $R=0$ depicts the throat; the curve $R= \pm 0.08$ corresponds to the outer surface. The blue area below the throat does not belong to the model, it does not describe any part of space-time. The dust shells begin and end their motion at singularities, presented by red broken lines. The upper unpainted area depicts the Reissner-Nordström space-time; the red horizontal line corresponds to the horizon; the red dot-dashed and dashed lines in the dust layer depict the apparent horizon, which is also the location of possible turning points for ingoing and outgoing light rays. The green, yellow, and blue curves correspond to propagation of photons, emitted from the shell $R=0.08$ at different times $\tau_{0}$, in the region $R>0$ (solid lines) and $R<0$ (dashed lines). Light propagation outside the dust layer is not shown.

In both figures, the gray area depicts the evolution of a dust layer between their initial and final singularities; actually, each curve $r(R, \tau)$ in this area, except $R=0$, corresponds to two shells, $R>0$ and $R<0$ ). The upper white areas in Figures 4 and 5 actually correspond to two copies of the outer Schwarzschild or Reissner-Nordström region, respectively. In both figures one can see photon trajectories that cross the whole dust layer in finite proper time $\tau$, which means that the wormhole parts of these black hole space-times are traversable.

\section{Conclusions}

In our recent work [56], we constructed collapsing dusty wormhole solutions using a special choice of arbitrary functions in Tolman's solution; then, the internal wormhole solution was matched to external Schwarzschild space-time regions. In the present paper, we considered the generalized Tolman ( $q$-Tolman) solution, describing the evolution of a spherical dust cloud in the presence of an external electric or magnetic field. The main results of this paper may be summarized as follows:

1. It has been shown that the $q$-Tolman dust clouds can contain wormhole throats under certain conditions on the arbitrary functions $f(R)$ and $F(R)$ of the general solution to the field equations.

2. It has been shown that throats can only exist in the elliptic branch of $q$-Tolman spacetimes and are in general located in T-regions. This means that if a dust layer is 
matched to external Reissner-Nordström or Schwarzschild space-time regions, the whole configuration is a black hole rather than a wormhole.

3. The $q$-Tolman space-times with throats are proven to exist for a finite period of time in their comoving reference frames. If $q=0$ (no electromagnetic field), the evolution takes place between two "spherical" singularities at which $r \rightarrow 0$, while at $q \neq 0$ the initial and final singularities are of shell-sticking nature $(d r / d R \rightarrow 0)$.

4. An analysis of radial null geodesics for particular examples of models under study has shown that the dust layers with throats can be traversable in both cases $q=0$ and $q \neq 0$. In other words, a photon can cross such a dust layer more rapidly than this layer collapses.

5. It has been shown that $q$-Tolman clouds with throats can form traversable wormholes in closed isotropic FRW cosmological models filled with dust-or, more precisely, connect two copies of such a model. Therefore, each of these universes, taken separately, preserves all its important physical features, including such an issue as a cosmological horizon. However, if the wormhole is large enough, one should also consider the view of "another universe" through this wormhole, which needs a separate study. Note also that these wormholes expand and contract together with the corresponding cosmological space-time.

Solutions with throats form a subfamily (satisfying the conditions (26)) in the whole set of $q$-Tolman solutions. A finite lifetime of models with throats, from one singularity to another, is an important feature of this family, while in general, with $q \neq 0$, nonsingular models are possible.

Author Contributions: Individual contributions of authors, K.A.B., P.E.K. and S.V.S. are equal and include the following: conceptualization, methodology, formal analysis, investigation, validation, visualization, writing - original draft preparation, review and editing. All authors have read and agreed to the published version of the manuscript.

Funding: P.E.K. and S.V.S. are supported by RSF grant No. 21-12-00130. Partially, this work was done in the framework of the Russian Government Program of Competitive Growth of the Kazan Federal University. K.A.B. was supported in part by the RUDN University Strategic Academic Leadership Program, by RFBR Project 19-02-00346, and by the Ministry of Science and Higher Education of the Russian Federation, Project "Fundamental properties of elementary particles and cosmology" N 0723-2020-0041.

Institutional Review Board Statement: Not applicable.

Informed Consent Statement: Not applicable.

Conflicts of Interest: The authors declare no conflict of interest.

\section{Note}

In some books we can find a value of $\mathcal{K}$ four times larger, for example [54]. However, one can verify that Equation (24) gives the correct result for the Friedmann solution (20) and (21): $\mathcal{K}=60 a_{0}^{2} / a^{6}$.

\section{References}

1. Flamm, L. Beitrage zur Einsteinschen Gravitationstheorie. Phys. Z. 1916, 17, 448.

2. Einstein, A.; Rosen, N. The particle problem in the General Theory of Relativity. Phys. Rev. 1935, 48, 73-77. [CrossRef]

3. Wheeler, J.A. Geons. Phys. Rev. 1955, 97, 511-536. [CrossRef]

4. Wheeler, J.A. Geometrodynamics; Academic Press: New York, NY, USA, 1962; 334p.

5. Bronnikov, K.A. Scalar-tensor theory and scalar charge. Acta Phys. Pol. B 1973, 4, 251.

6. Ellis, H.G. Ether flow through a drainhole-A particle model in general relativity. J. Math. Phys. 1973, 14, 104-118. [CrossRef]

7. Ellis, H.G. The evolving, flowless drainhole: A nongravitating-particle model in general relativity theory. Gen. Relativ. Gravit. 1979, 10, 105-123. [CrossRef]

8. Clément, G. A class of wormhole solutions to higher dimensional general relativity. Gen. Relativ. Gravit. 1984, 16, 131-138. [CrossRef]

9. Clément, G. Axisymmetric regular multiwormhole solutions in five-dimensional general relativity. Gen. Relativ. Gravit. 1984, 16, 477-489. [CrossRef] 
10. Morris, M.S.; Thorne, K.S. Wormholes in spacetime and their use for interstellar travel: A tool for teaching general relativity. Am. J. Phys. 1988, 56, 395. [CrossRef]

11. Visser, M. Lorentzian Wormholes: From Einstein to Hawking; American Institute of Physics: Woodbury, NY, USA, 1995; 412p.

12. Lobo, F.S.N. Exotic solutions in General Relativity: Traversable wormholes and "warp drive" spacetimes. In Classical and Quantum Gravity Research; Nova Science Publishers: New York, NY, USA, 2008; pp. 1-78.

13. Bronnikov, K.A. Spherically symmetric solutions in D-dimensional dilaton gravity. Gravit. Cosmol. 1995, 1, 67-78.

14. Clément, G.; Fabris, J.C.; Rodrigues, M.E. Phantom black holes in Einstein-Maxwell-dilaton theory. Phys. Rev. D 2009, 79, 064021. [CrossRef]

15. Goulart, P. Phantom wormholes in Einstein-Maxwell-dilaton theory. Class. Quantum Gravity 2017, 35, 025012. [CrossRef]

16. Huang, H.; Yang, J. Charged Ellis wormhole and black bounce. Phys. Rev. D. 2019, 100, 124063. [CrossRef]

17. Yang, J.; Huang, H. Trapping Horizons of the Evolving Charged Wormhole and Black Bounce. arXiv 2021, arXiv:2104.11134.

18. Matos, T.; Miranda G. Exact rotating magnetic traversable wormhole satisfying the energy conditions. Phys. Rev. D 2019, 99, 124045.

19. Blázquez-Salcedo, J.L; Knoll, C.; Radu, E. Traversable wormholes in Einstein-Dirac-Maxwell theory. Phys. Rev. Lett. 2021, 126, 101102. [CrossRef] [PubMed]

20. Konoplya, R.A.; Zhidenko, A. Traversable wormholes in general relativity without exotic matter. arXiv 2021, arXiv:2106.05034.

21. Bolokhov, S.V.; Bronnikov, K.A.; Krasnikov, S.V.; Skvortsova, M.V. A note on “Traversable wormholes in Einstein-Dirac-Maxwell theory". arXiv 2021, arXiv:2104.10933.

22. Alencar, G.; Nilton, M. Schwarzschild-like wormholes in asymptotically safe gravity. Universe 2021, 7, 332.

23. Lobo, F.S.N. Chaplygin traversable wormholes. Phys. Rev. D 2006, 73, 064028. [CrossRef]

24. Bronnikov, K.A.; Baleevskikh, K.A.; Skvortsova, M.V. Wormholes with fluid sources: A no-go theorem and new examples. Phys. Rev. D 2017, 96, 124039. [CrossRef]

25. Sushkov, S.V. Wormholes supported by a phantom energy. Phys. Rev. D, 2005, 71, 043520. [CrossRef]

26. Kuhfittig, P.K.F. Conformal-symmetry wormholes supported by a perfect fluid. New Horizons Math. Phys. 2017, 1, 14-18. [CrossRef]

27. Lobo, F.S.N. Stable phantom energy traversable wormhole models. AIP Conf. Proc. 2006, 861, 936-943.

28. Kuhfittig, P.K.F. Exactly solvable wormhole and cosmological models with a barotropic equation of state. Acta Phys. Pol. B 2016, 47, 1263-1272. [CrossRef]

29. Sahoo, P.K.; Moraes, P.H.R.S.; Sahoo, P.G.R. Phantom fluid supporting traversable wormholes in alternative gravity with extra material terms. Int. J. Mod. Phys. D 2018, 27, 1950004. [CrossRef]

30. Kuhfittig, P.K.F. Static and dynamic traversable wormhole geometries satisfying the Ford-Roman constraints. Phys. Rev. D 2002, 66, 024015. [CrossRef]

31. Visser, M. Traversable wormholes: Some simple examples. Phys. Rev. D 1989, 39, 3182. [CrossRef]

32. Visser, M. Traversable wormholes from surgically modified Schwarzschild spacetimes. Nucl. Phys. B 1989, 328, 203-212. [CrossRef]

33. Kar, S. Evolving wormholes and the energy conditions. Phys. Rev. D 1994, 49, 862. [CrossRef]

34. Kim, S.W. Cosmological model with a traversable wormhole. Phys. Rev. D 1996, 53, 6889. [CrossRef]

35. Roman, T.A. Inflating Lorentzian wormholes. Phys. Rev. D 1993, 47, 1370-1379. [CrossRef]

36. Sushkov, S.V.; Kim, S.W. Cosmological evolution of a ghost scalar field. Gen. Relativ. Gravit. 2004, 36, 1671-1678. [CrossRef]

37. Sushkov, S.V.; Zhang, Y.Z. Scalar wormholes in cosmological setting and their instability. Phys. Rev. D 2008, 77, 024042. [CrossRef]

38. Wang, A.; Letelier, P.S. Dynamic wormholes and energy conditions. Prog. Theor. Phys. 1995, 94, 137-142. [CrossRef]

39. Hayward, S.A. Wormhole dynamics in spherical symmetry. Phys. Rev. D 2009, 79, 124001. [CrossRef]

40. Hochberg, D.; Visser, M. Dynamic wormholes, anti-trapped surfaces, and energy conditions. Phys. Rev. D 1998, 58, 04402. [CrossRef]

41. Hochberg, D.; Visser, M. Geometric structure of the generic static traversable wormhole throat. Phys. Rev. D 1997, 56, 4745. [CrossRef]

42. Bronnikov, K.A.; Starobinsky, A.A. No realistic wormholes from ghost-free scalar-tensor phantom dark energy. JETP Lett. 2007, 85, 1-5. [CrossRef]

43. Bronnikov, K.A. Krechet, V.G. Potentially observable cylindrical wormholes without exotic matter in GR. Phys. Rev. D 2019, 99, 084051. [CrossRef]

44. Bolokhov, S.V.; Bronnikov, K.A.; Skvortsova, M.V. Cylindrical wormholes: A search for viable phantom-free models in GR. Int. J. Mod. Phys. D 2019, 28, 1941008.

45. Bronnikov, K.A.; Krechet, V.G.; Oshurko, V.B. Rotating Melvin-like universes and wormholes in general relativity. Symmetry 2020, 12, 1306. [CrossRef]

46. Bronnikov, K.A.; Galiakhmetov, A.M. Wormholes without exotic matter in Einstein-Cartan theory. Gravit. Cosmol. 2015, 21, 283-288. [CrossRef]

47. Bronnikov, K.A.; Galiakhmetov, A.M. Wormholes and black universes without phantom fields in Einstein-Cartan theory. Phys. Rev. D 2016, 94, 124006. [CrossRef]

48. Arellano, A.V.B.; Lobo, F.S.N. Evolving wormhole geometries within nonlinear electrodynamics. Class. Quantum Gravity 2006, 23, 5811-5824. [CrossRef]

49. Bronnikov, K.A. Nonlinear electrodynamics, regular black holes and wormholes. Int. J. Mod. Phys. D 2018, 27, 184105. [CrossRef]

50. Tolman, R. Effect of inhomogeneity on cosmological models. Proc. Natl. Acad. Sci. USA 1934, 20, 169-176. [CrossRef] 
51. Lemaître, G. L'Univers en expansion. Ann. Soc. Sci. Brux. 1933, A53, 51-85.

52. Bondi, H. Spherically symmetrical models in general relativity. Mon. Not. R. Astron. Soc. 1947, 107, 410; Reprinted in Gen. Relativ. Gravit. 1999, 31, 1783-1805. [CrossRef]

53. Landau, L.D.; Lifshitz, E.M. The Classical Theory of Fields, 4th ed.; Butterworth-Heinemann: Oxford, UK, 1987; Volume 2, 402p.

54. Bambi, C. Black Holes: A Laboratory for Testing Strong Gravity; Springer Nature Singapore Pte Ltd.: Singapore, 2017; 355p.

55. Christodoulou, D. Violation of cosmic censorship in the gravitational collapse of a dust cloud. Commun. Math. Phys. 1984, 93, 171-195. [CrossRef]

56. Kashargin, P.; Sushkov, S. Collapsing wormholes sustained by dustlike matter. Universe 2020, 6, 186. [CrossRef]

57. Markov, M.A.; Frolov, V.P. Metrics of the closed Friedman world perturbed by electric charge (to the theory of electromagnetic «Friedmons»). Teor. Mat. Fiz. 1970, 3, 3-17.

58. Bailyn, M. Oscillatory behavior of charge-matter fluids with $e / m>G^{1 / 2}$. Phys. Rev. D 1973, 8, 1036. [CrossRef]

59. Vickers, P.A. Charged dust spheres in general relativity. Ann. Inst. Henri Poincaré A 1973, 18, 137.

60. Ivanenko, D.D.; Krechet, V.G.; Lapchinskii, V.G. The dynamics of charged dust in the general theory of relativity. Sov. Phys. J. 1973, 16, 1675-1679. [CrossRef]

61. Khlestkov, Y.A. Three types of solutions of the Einstein-Maxwell equations. J. Exp. Teor. Fis. 1975, 41, 188.

62. Shikin, I.S. An investigation of a class of gravitational fields for a charged dustlike medium. J. Exp. Teor. Fis. 1975, 40, 215.

63. Pavlov, N.V. Charged dust spheres in the general theory of relativity I. Quadratures of Einstein's equations. Sov. Phys. J. 1976, 19, 489-495. [CrossRef]

64. Pavlov, N.V.; Bronnikov, K.A. Charged dust spheres in the general theory of relativity II. Singularities and physically permissible models. Sov. Phys. J. 1976, 19, 916-920. [CrossRef]

65. Bronnikov K.A.; Kovalchuk, M.A. Some exact models for nonspherical collapse, I. Gen. Rel. Grav. 1983, 15, 809-822. [CrossRef]

66. Bronnikov K.A.; Kovalchuk, M.A. Some exact models for nonspherical collapse, II. Gen. Rel. Grav. 1983, 15, 823-836. [CrossRef]

67. Bronnikov K.A.; Kovalchuk, M.A. Some exact models for nonspherical collapse, III. Gen. Rel. Grav. 1984, 16, 15-31. [CrossRef]

68. Shatskiy, A.A.; Novikov, I.D.; Kardashev, N.S. A dynamic model of the wormhole and the Multiverse model. Uspekhi Fiz. Nauk 2008, 178, 481-488. [CrossRef]

69. Khlestkov, Y.A.; Sukhanova, L.A. Internal structure of wormholes-Geometric images of charged particles in general relativity. Gravit. Cosmol. 2018, 24, 360-370. [CrossRef]

70. Tomikawa, Y.; Izumi, K.; Shiromizu, T. New definition of a wormhole throat. Phys. Rev. D 2015, 91, 104008. [CrossRef]

71. Bittencourt, E.; Klippert, R.; Santos, G. Dynamical wormhole definitions confronted. Class. Quantum Gravity 2018, $35,155009$. [CrossRef]

72. Reissner, H. Über die Eigengravitation des elektrischen Feldes nach der einsteinschen. Ann. Phys. 1916, 355, 106-120. [CrossRef]

73. Nordström, G. On the energy of the gravitational field in Einstein's theory. Proc. Kon. Ned. Akad. Wet. 1918, 20, $1238-1245$.

74. Hawking, S.W.; Ellis, G.F.R. The Large Scale Structure of Space-Time; Cambridge UP: Cambridge, UK, 1973; 391p.

75. Darmois, G. Les équations de la gravitation einsteinienne. In Mémorial des Sciences Mathematiques; Gauthier-Villars: Paris, France, 1927; Volume 25.

76. Israel, W. Singular hypersurfaces and thin shells in general relativity. Nuovo Cim. B 1967, 48, 463. [CrossRef] 